\title{
A Rare Case of Isolated Cerebral Sarcoidosis Presenting as Suprasellar Mass Lesion with Salt- Wasting Hypopituitarism
}
H. Krenzlin ${ }^{1}$
D. Jussen ${ }^{1}$
C. Musahl ${ }^{1}$
S. Scheil-Bertram²
K. Wernecke ${ }^{3}$
P. Horn ${ }^{1}$

${ }^{1}$ Department of Neurosurgery, Dr. Horst Schmidt Kliniken, HELIOS Klinikum, Wiesbaden, Germany

2 Department of Pathology and Cytology, Dr. Horst Schmidt Kliniken, HELIOS Klinikum, Wiesbaden, Germany

${ }^{3}$ Department of Radiology and Radiotherapy (RNS), Dr. Horst Schmidt

Address for correspondence $\mathrm{H}$. Krenzlin, MD, Klinik für Neurochirurgie, Dr. Horst-Schmidt-Kliniken, Wiesbaden, LudwigErhard-Strasse 100, 65199 Wiesbaden, Germany

Kliniken, Wiesbaden, Germany (e-mail: harald.krenzlin@hsk-wiesbaden.de).

J Neurol Surg Rep 2015;76:e140-e145.

\begin{abstract}
Background Sarcoidosis is a systemic disorder of unknown origin characterized by noncaseating granulomas. Clinical symptoms due to central nervous system (CNS) involvement occur in 5 to $7 \%$ of all cases; subclinical involvement is more frequent. Sole CNS involvement is very rare.

Case Report A 25-year-old man presented with increasing polyuria and polydipsia over 8 weeks. Magnetic resonance imaging (MRI) revealed a supra- and infra-chiasmatic pre-

Keywords

- neurosarcoidosis

- intracranial mass lesion

- salt-wasting hypopituitarism

- diabetes insipidus thalamic mass lesion $1.0 \times 1.4 \times 1.4 \mathrm{~cm}$ in diameter. Microsurgical biopsy verified a necrotizing noncaseating epithelioid cell tumor indicative for neurosarcoidosis. All symptoms dissolved within 3 months under stringent corticoid therapy.

Conclusion Intracranial mass lesions as the primary and only manifestation of neuronal sarcoidosis are rare. Because conservative treatment is safe and effective, surgery is limited to biopsy and the alleviation of pressure-related symptoms to preserve neurologic function.
\end{abstract}

\section{Introduction}

Sarcoidosis is a systemic granulomatous disease whose etiology is still unknown. The incidence is estimated to be as high as 20 per $100,000 .{ }^{1}$ Only a few patients develop a neurologic involvement throughout the course of their disease. A manifestation limited to the central nervous system (CNS) is rare. Due to the absence of any specific criteria, the diagnosis is often difficult and delayed. We present a quintessential case of isolated intracranial sarcoidosis mimicking a suprasellar mass lesion causing hypophyseal insufficiency.

\section{Case Report}

\section{History}

A 25-year-old white man presented with increasing polyuria and polydipsia over 8 weeks. Daily fluid intake reached $12 \mathrm{~L}$ per day. In addition, the patient complained about sweating, fatigue, and impaired heat tolerance. He denied fever or current infections. Medical history revealed a chronic thyroxin-dependent hypothyroidism. Social anamnesis and family history were normal.

\section{Physical Examination}

His body weight was $85 \mathrm{~kg}$ and his height was $174 \mathrm{~cm}$ (body mass index: 28.1). On inspection the skin was pale and sweaty. Pitting edemas were palpable on both lower limbs. Body temperature was $37.2^{\circ} \mathrm{C}$. Blood pressure was $130 / 80$. Normal heart sounds were heard on auscultation. Peripheral pulses were all palpable, strong, and regular at a heart rate of $80 \mathrm{bpm}$; no jugular venous pressure (JVP) elevation was visible. No enlarged lymph nodes were palpable. The abdomen was soft with no masses. On neurologic examination the patient was oriented. His muscle tone was normal; no paresis
License terms

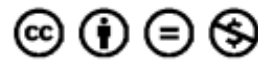

received

June 22, 2014 accepted after revision

February 14, 2015

published online

April 27, 2015
DOI http://dx.doi.org/ 10.1055/s-0035-1549310. ISSN 2193-6366.

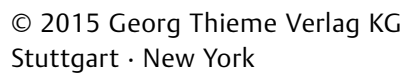


was present or sensory deficits. All joints were painless with a normal range of motion. There were no signs of nuchal rigidity. Tendon reflexes were normal. No deficits of eyesight or visual field were found on ophthalmologic examination.

\section{Laboratory Work-up}

Blood tests showed a hyponatremia of $127 \mathrm{mmol} / \mathrm{L}$ (136-146 $\mathrm{mmol} / \mathrm{L}$ ). As a marker for sarcoidosis, angiotensin-converting enzyme (ACE) was slightly decreased at $18 \mathrm{U} / \mathrm{L}(20-70 \mathrm{U} / \mathrm{L})$. The soluble interleukin- $\mathrm{R}$ receptor was elevated at $868 \mathrm{U} / \mathrm{L}$ (158-623 $\mathrm{U} / \mathrm{L})$. Prolactin levels were within normal range at $4.5 \mu \mathrm{g} / \mathrm{L}(4-15$ $\mu \mathrm{g} / \mathrm{L})$. Diagnostic lumbar puncture revealed no abnormalities. Urine specific gravity was lowered to 1.010 (1.020-1.025).

\section{Imaging}

A chest X-ray performed on admission showed no abnormalities.

\section{MRI Imaging}

T2-weighted cranial magnetic resonance imaging (MRI) images revealed a supra- and infra-chiasmatic pre-thalamic mass lesion $1 \times 1.4 \times 1.4 \mathrm{~cm}$ in diameter, extending from the lamina terminalis into the third ventricle. The lesion appeared solid, fuzzy, and T2-hyperintens. No leptomeningeal involvement was noted. The inhomogeneous wall stretched filamentous to the surrounding tissue. After administration of intravenous gadolinium contrast, a strong signal enhancement was noted (-Fig. 1).

\section{Differential Diagnosis}

Possible pathologic conditions causing suprasellar mass lesions encompass entities such as pituitary adenomas and carcinomas, pituitary pseudotumors, vascular lesions, craniopharyngiomas, meningiomas, germ cell tumors, hypothalamic and optic gliomas, lymphoma, metastasis, epidermoid cysts, tuberculosis, and cysticercosis as well as inflammatory diseases such as sarcoidosis or lymphocytic adenohypophysitis.

\section{Biopsy}

Based on clinical and imaging findings, microsurgery was initiated to establish the diagnosis based on histopathology and perform a tumor resection if feasible. Surgery was performed using a subfrontal approach. After a subdural cisternostomy, the optic nerve and chiasm were exposed. The arachnoid layer covering the lamina terminalis was carefully dissected, freeing a curly flower-like exophytic lesion. The lesion was excavated toward the third ventricle. Multiple specimens were sent for intrasurgical frozen section. Immediate pathology suggested a necrotizing epithelioid cell tumor indicative of chronic inflammation. Thus the surgical exploration was confined at this stage (-Fig. 2).

Further intra- and postoperative course was uneventful. No new neurologic deficits were detected. Postoperative MRI showed no signs of ischemia or bleeding.

\section{Histopathology}

Definite histology proved epithelioid cell granulomas and multinucleated giant cells with surrounding lymphocytes. Occasional central necroses without caseation were noted. Granulomas were rich in fibrous tissue and reticulin. PAS and Ziehl-Neelsen staining were negative. The polymerase chain reaction remained negative for mycobacteria (-Fig. $\mathbf{3}$ ).

\section{Treatment}

After histopathologic confirmation, a glucocorticoid therapy using dexamethasone $8 \mathrm{mg}$ three times a day was initiated. Subsequently the fluid uptake attenuated to normal amounts. After 8 weeks it had decreased to 4 L per day with consecutive normouria. The hyponatremia normalized to $4.5 \mathrm{mmol} / \mathrm{L}$. The patient developed signs of Cushing disease and complained about painfully swollen joints. All symptoms vanished after reduction of the corticosteroid treatment.

In MRI scans 3 months after admission the preexisting mass lesion had regressed completely. Only the pituitary stalk showed a residual, limited focal contrast enhancement in T1-weighted images after gadolinium administration ( - Fig. 4).

\section{Discussion}

Sarcoidosis, also known as Boeck or Schaumann disease, is a systemic disorder defined by the presence of noncaseating granulomas that compromise the integrity and function of any tissue where they occur. Chronic as well as self-limiting forms are described. ${ }^{2}$ Sarcoidosis is histopathologic characterized by the presence of noncaseating epithelioid-cell granulomas in several
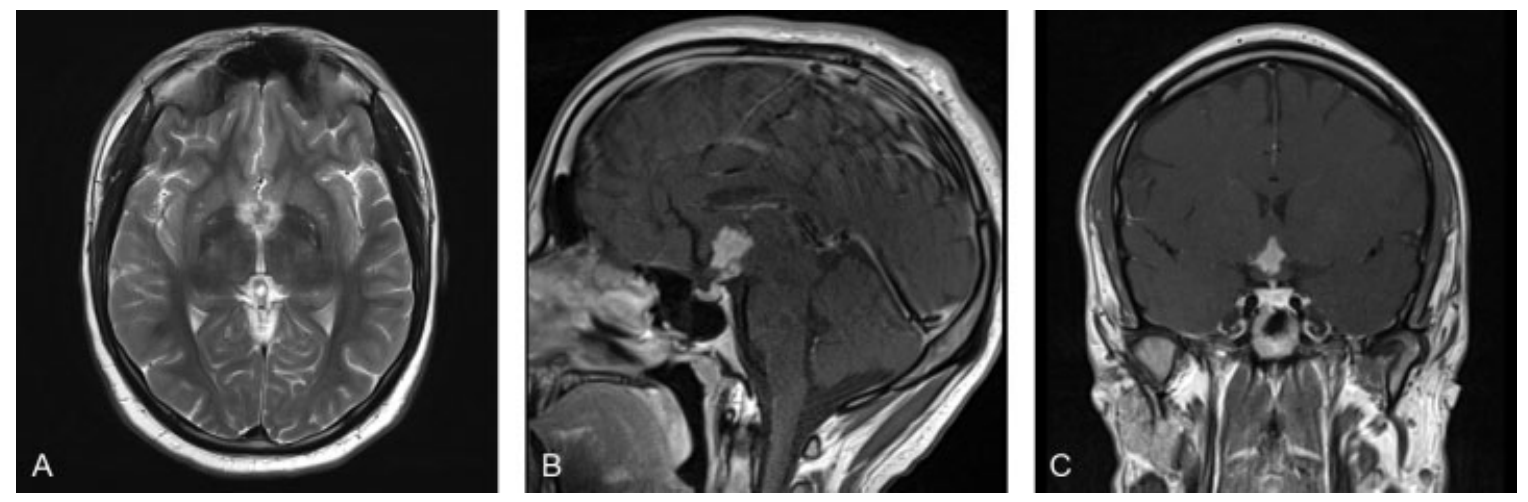

Fig. 1 Preoperative magnetic resonance imaging. (A) T2-weighted image depicts a $1.8 \times 1.2 \mathrm{~cm}$ hypointense filamentous spreading suprasellar mass lesion above the optic chiasm. (B and C) T1-weighted imaging after intravenous gadolinium: inhomogeneous contrast enhancement with contact of the lesion to the pituitary stalk. 

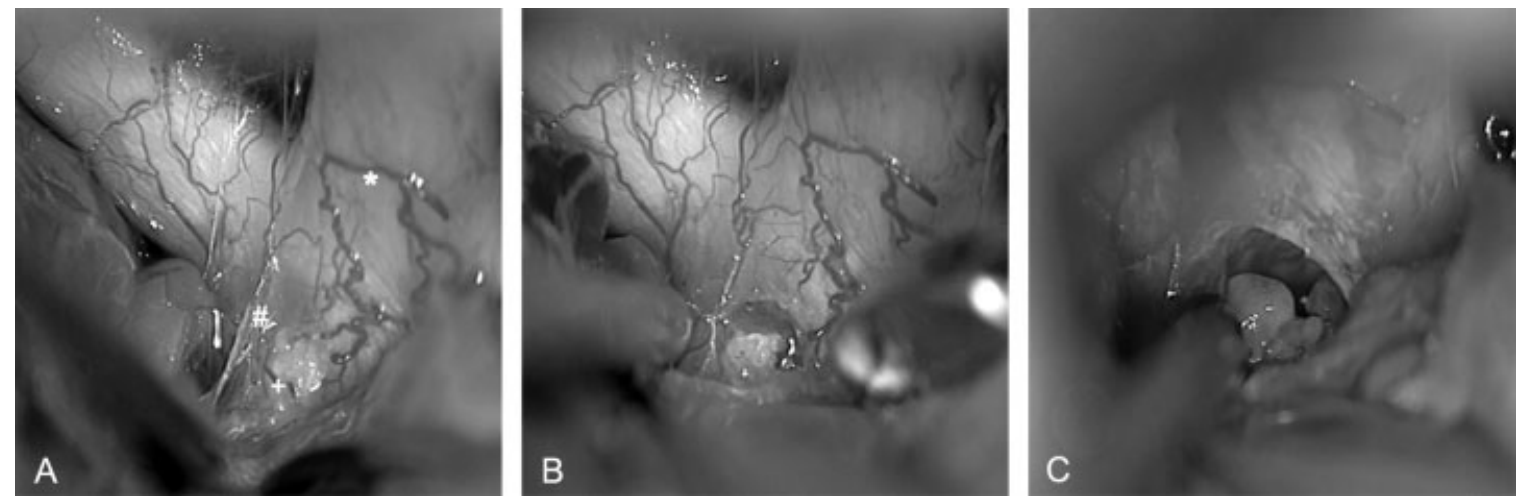

Fig. 2 Intraoperative aspect. (A) Suprasellar mass lesion, optic nerve (asterisk) with arachnoid, and curly flower-like lesion (plus sign) arising from the lamina terminalis (number sign). (B) Opening of the arachnoid mater. (C) Aspect after repeated biopsy.

affected organs. Involvement of only one organ or compartment is less common and only reported sporadically. The multiple lesions do not contain tuberculosis-like necrotizing areas or mycobacterium tuberculosis. Some may develop fibrinoid or paramyeloid necrosis. Epithelioid or multinucleated cell formation occurs in differing frequency. These cells contain nonspecific asteroid or radiating inclusion bodies, so-called Schaumann bodies. Granuloma progression either leads to resolution or conversion into hyaline connective tissue.

The first CNS form was described as early as 1905 when Jörgen Schaumann recognized the systemic nature and genetical equity of Boeck sarcoid and lupus pernio. Later osseous forms were described. Due to its highly variable clinical appearance, the incidence of sarcoidosis can only be estimated from mass screenings and is assumed to be as high as 20 per $100,000 .^{1}$ Sarcoidosis is most frequent in young adults with a female predominance. ${ }^{2}$ Only $16 \%$ of all cases develop clinical symptoms. Neurologic symptoms due to CNS involvement are found in 5 to $7 \%$ of all patients with systemic sarcoidosis. ${ }^{3-5}$ The number of silent neural signs detected solely by autopsy is even higher. ${ }^{6}$ Rarely sarcoidosis is strictly confined to CNS manifestations with an estimated incidence $<0.2$ per 100,000 within the white population. ${ }^{5-7}$ Lesions found in CNS tissue are no different from those found in other organs. Spreading occurs leptomeningeally, alongside the Virchow-Robin spaces and entering the cortical and subcortical layers following penetrating blood vessels. Thus the hypothalamus, third ventricle, pituitary gland, optic nerve, and chiasm are most frequently involved. There is a broad variety of clinical variants with intra- or extraaxial diffusely disseminated or more localized leptomeningeal or parenchymal plaques reported. ${ }^{2,8,9}$ In 1947 , Everts et al described the first tumorlike CNS lesion caused by nonsystemic sarcoidosis. Clinical symptoms depend on location of the lesion and encompass seizures, sensor and motor deficits, pituitary dysfunction, neuropsychological deficits, and cranial nerve palsies as well as hydrocephalus and unspecific headaches. ${ }^{10-14}$

In MR tomography, neurosarcoidosis lesions present as nodular or diffusely infiltrating that are mostly attached to the leptomeninges. After intravenous administration of gadoliniumdiethylenetriamine pentaacetic acid, a homogeneous or diffuse enhancement has been reported. MRI is the imaging modality of choice. ${ }^{15}$ Without systemic spread and symptoms, neurosarcoidosis can mimic almost every other intracranial disease in clinical and imaging appearance. Therefore the diagnostic process is often prolonged and challenging.

In this case report, we presented a patient with isolated cerebral sarcoidosis presenting with isolated diabetes insipidus. The initial MRI scan revealed a T2 hyperintense supra- and infrachiasmatic mass lesion with inhomogeneous enhancement after intravenous gadolinium administration. The imaging results fall in line with the described pattern commonly observed in intracranial neurosarcoidosis. Nevertheless there is a whole
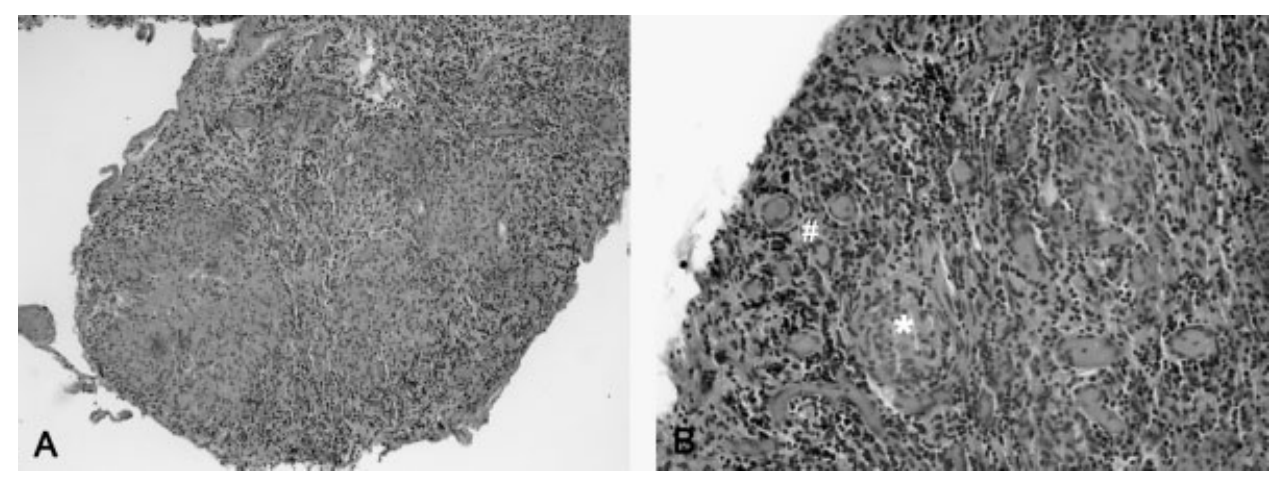

Fig. 3 Microphotographs of the histopathologic specimen stained with hematoxylin and eosin. (A) Overview, original magnification $\times 10$. (B) Noncaseating epithelioid cell granulomas (asterisk) and multinuclear giant cells (number sign); original magnification $\times 20$. 

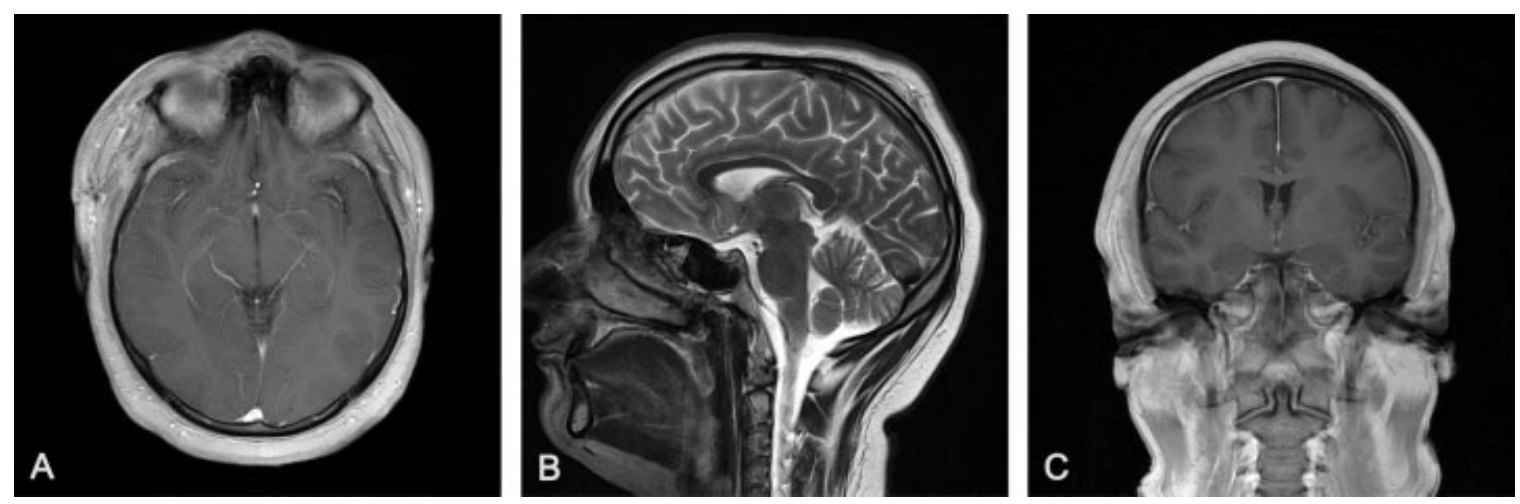

Fig. 4 Postoperative magnetic resonance imaging. (A and C) T1-weighted images after intravenous contrast and (B) T2-weighted image depicting complete tumor regression with no residual gadolinum enhancement.

plethora of more commonly seen differential diagnoses presenting with similar tomographic findings. Extensive blood tests and cerebrospinal fluid (CSF) examination revealed no findings indicating the presence of neurosarcoidosis. ACE can be of diagnostic value because elevation indicates the presence of neurosarcoidosis. The low sensitivity of ACE elevation in the cerebrospinal fluid (CSF) leads to a high number of false-negative results. ${ }^{16,17}$ Although distinction of neurosarcoidosis from other tumor entities by CSF examination alone is impossible, elevation of serum ACE occurs in 56 to $85 \%$ of all cases of sarcoidosis. ${ }^{17,18}$ Thus ACE elevation is indicative but may be observed in other inflammatory diseases such as multiple sclerosis, Behçet disease, Guillain-Barré syndrome, and other infections. The ACE level in the presented case was normal in serum and in CSF, illustrating the low sensitivity and specificity for the diagnosis of neurosarcoidosis. After completion of the diagnostic work-up, an intracranial neoplasia such as a germinoma or craniopharyngioma seemed the most likely diagnosis. Although not overly common in western European countries, cerebral tuberculosis should always be considered because radiography and histopathology share numerous similarities. To initiate adequate therapy, histopathologic diagnosis is essential. We therefor pursued a microsurgical approach that could be used for radical tumor removal if feasible. Only open microsurgical biopsy was able to establish the diagnosis in the current case.

Over the last decade, several case reports have been published depicting intracranial lesions in patients with known sarcoidosis. Primary neurosarcoidosis in the absence of a systemic disease is still diagnostically a headache. Pituitary involvement occurs in $10 \%$ of all patients with neurosarcoidosis. ${ }^{19}$ Patients often present with profound endocrinological dysfunction including obesity, psychosis, diabetes insipidus, amenorrhea, and hypoglycemia. ${ }^{20}$ Symptoms may be secondary to pituitary or thalamic infiltration and destruction..$^{21}$ Diabetes insipidus occurrence is attributed to an infiltration of the pituitary stalk.

Thus the presented case illustrates a rare manifestation of neurosarcoidosis and demonstrates the diagnostic obstacles and pitfalls of this disease. Several case reports dealing with the diagnostically and therapeutically conundrum have been published over the last decade. Guoth et al describe a young woman with clinical hypopituitarism and systemic sarcoidosis of the lung, gastrointestinal tract, and a cystic pituitary mass. ${ }^{21}$ Isolated CNS involvement mimicking a mass lesion without disseminated disease or prior systemic appearance is even more rare. Grand et al report a pseudotumoral right frontotemporal mass lesion as only sign of the disease. ${ }^{12}$ Another case was presented by Stübgen describing a 22-year-old woman with a clival mass as the only manifestation. ${ }^{22}$-Table 1 lists the cases published in the English literature over the last decade. In most cases signs of a systemic disease were apparent at the time of pituitary-hypothalamic symptom development. In these cases a conservative approach with corticosteroid treatment is feasible. Histopathology should be established via bronchial lavage or lymph node biopsy. In cases of primary solitary intracranial mass lesions, a trial corticosteroid treatment should be considered in stationary diseases. In patients presenting with pressure-related symptoms or signs of fast disease progression, microsurgery is indicated.

\section{Conclusion}

Neuronal sarcoidosis mimicking intracranial mass lesions has been described in several case reports over the past decade. In most of theses cases systemic manifestations preceded or accompanied the neuronal manifestation. Almost all localizations of intracranial lesions have been described including sellar and suprasellar lesions. More than one intracranial lesion is found in most of the patients. A solitary sellar mass lesion due to neurosarcoidosis has only mentioned twice to our knowledge. . $^{2425}$

CSF examination with pleocytosis, ACE- and proteinlevel elevation is indicative but not conclusive for the diagnosis of neurosarcoidosis. Therefor biopsy taking is still obligatory today. There is no evidence of a superior clinical benefit from surgical resection because neurosarcoid lesions respond well to steroid therapy. ${ }^{26}$ Surgery should therefore be confined to the collection of biopsy material as soon as histopathology is available. In cases of clinically apparent lesions, microsurgery should be considered to alleviate pressure-related symptoms or deficits caused by space occupation alone. 


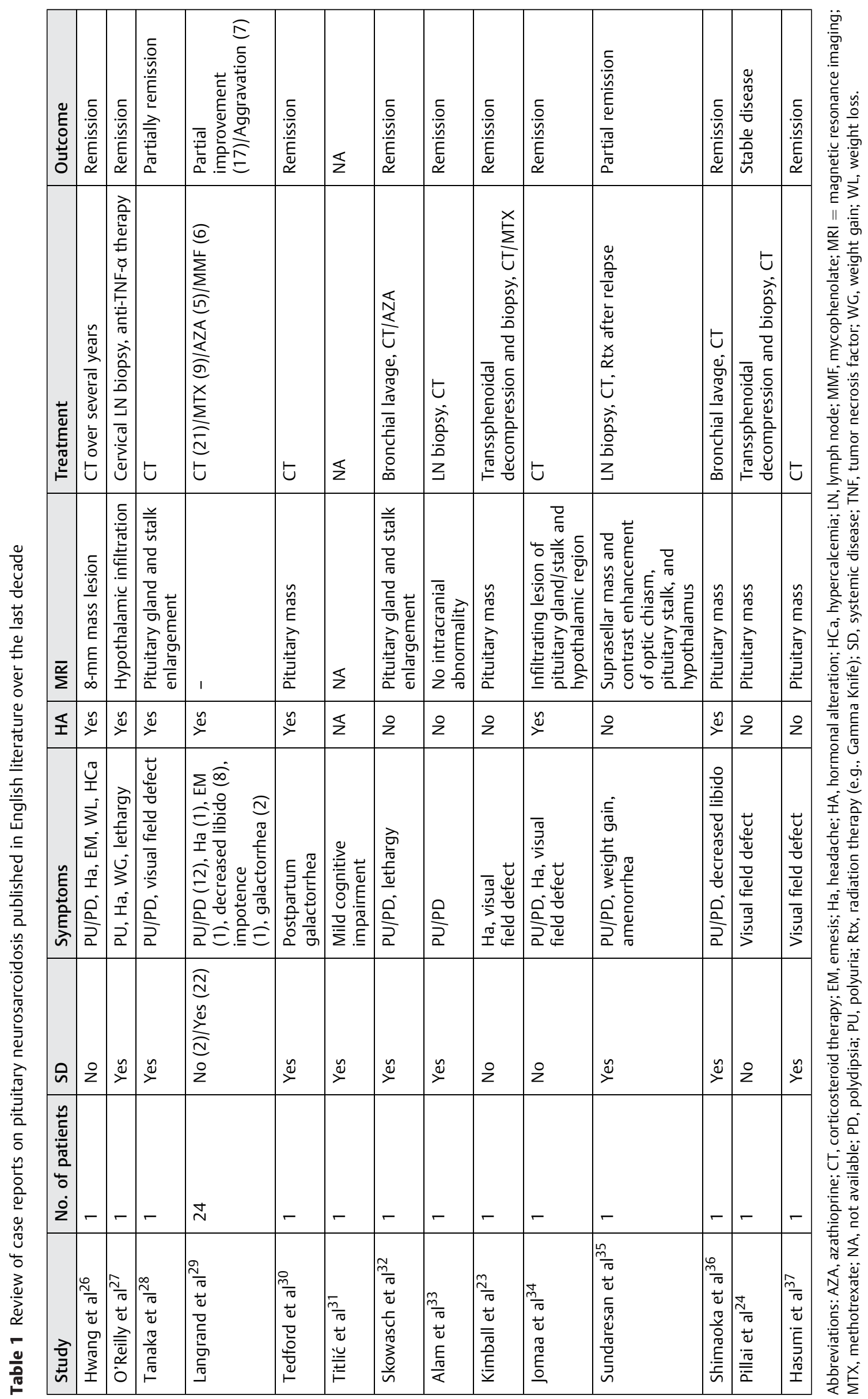




\section{References}

1 James DG, Neville E, Carstairs LS. Bone and joint sarcoidosis. Semin Arthritis Rheum 1976;6(1):53-81

2 Lexa FJ, Grossman RI. MR of sarcoidosis in the head and spine: spectrum of manifestations and radiographic response to steroid therapy. AJNR Am J Neuroradiol 1994;15(5):973-982

3 Mayock RL, Bertrand P, Morrison CE, Scott JH. Manifestations of sarcoidosis: analysis of 145 patients, with a review of nine series selected from the literature. Am J Med 1963;35(1):67-89

4 James DG, Neville E, Siltzbach LE. A worldwide review of sarcoidosis. Ann N Y Acad Sci 1976;278(1):321-334

5 Stern BJ, Krumholz A, Johns C, Scott P, Nissim J. Sarcoidosis and its neurological manifestations. Arch Neurol 1985;42(9):909-917

6 Ricker W, Clark M. Sarcoidosis; a clinicopathologic review of 300 cases, including 22 autopsies. Am J Clin Pathol 1949;19(8): 725-749

7 Chen RC, McLeod JG. Neurological complications of sarcoidosis. Clin Exp Neurol 1989;26:99-112

8 Christoforidis GA, Spickler EM, Recio MV, Mehta BM. MR of CNS sarcoidosis: correlation of imaging features to clinical symptoms and response to treatment. AJNR Am J Neuroradiol 1999;20(4): 655-669

9 Clark WC, Acker JD, Dohan FC Jr, Robertson JH. Presentation of central nervous system sarcoidosis as intracranial tumors. J Neurosurg 1985;63(6):851-856

10 Berek K, Kiechl S, Willeit J, Birbamer G, Vogl G, Schmutzhard E. Subarachnoid hemorrhage as presenting feature of isolated neurosarcoidosis. Clin Investig 1993;71(1):54-56

11 Brooks ML, Wang AM, Black PM, Haikal N. Subdural mass lesion secondary to sarcoid granuloma MR and CT findings and differential diagnosis. Comput Med Imaging Graph 1989;13(2):199-205

12 Grand S, Hoffmann D, Bost F, Francois-Joubert A, Pasquier B, Le Bas JF. Case report: pseudotumoral brain lesion as the presenting feature of sarcoidosis. Br J Radiol 1996;69(819):272-275

13 Larner AJ, Ball JA, Howard RS. Sarcoid tumour: continuing diagnostic problems in the MRI era. J Neurol Neurosurg Psychiatry 1999;66(4):510-512

14 Mayer SA, Yim GK, Onesti ST, Lynch T, Faust PL, Marder K. Biopsyproven isolated sarcoid meningitis. Case report. J Neurosurg 1993; 78(6):994-996

15 Leu NH, Chen CY, Wu CS, Lee KW, Hsiao HS, Zimmerman RA. Primary chiasmal sarcoid granuloma: MRI. Neuroradiology 1999; 41(6):440-442

16 Tahmoush AJ, Amir MS, Connor WW, et al. CSF-ACE activity in probable CNS neurosarcoidosis. Sarcoidosis Vasc Diffuse Lung Dis 2002;19(3):191-197

17 Jones DB, Mitchell D, Horn DB, Edwards CR. Cerebrospinal fluid angiotensin converting enzyme levels in the diagnosis of neurosarcoidosis. Scott Med J 1991;36(5):144-145

18 Scott TF. Neurosarcoidosis: progress and clinical aspects. Neurology 1993;43(1):8-12

19 Arle JE, Judkins AR, Kotapka MJ. Neurosarcoidosis presenting in the pituitary gland with normal endocrine studies. Skull Base Surg 1999;9(2):149-153
20 Smith D, Cullen MJ. Two cases of hypothalamic-pituitary sarcoidosis. J R Soc Med 2005;98(4):167-169

21 Guoth MS, Kim J, de Lotbiniere AC, Brines ML. Neurosarcoidosis presenting as hypopituitarism and a cystic pituitary mass. Am J Med Sci 1998;315(3):220-224

22 Stübgen JP. Neurosarcoidosis presenting as a retroclival mass. Surg Neurol 1995;43(1):85-87; discussion 87-88

23 Kimball MM, Wind JJ, Codispoti KE, Jones RV, Leiphart JW. Neurosarcoidosis presenting as an isolated intrasellar mass: case report and review of the literature. Clin Neuropathol 2010;29(3): 156-162

24 Pillai P, Ray-Chaudhury A, Ammirati M, Chiocca EA. Solitary pituitary sarcoidosis with normal endocrine function: case report. J Neurosurg 2008;108(3):591-594

25 Oksanen V. Neurosarcoidosis: clinical presentations and course in 50 patients. Acta Neurol Scand 1986;73(3):283-290

26 Hwang JK, Cho JH, Park SY, et al. A case of possible neurosarcoidosis presenting as intractable headache and panhypopituitarism. Case Rep Endocrinol 2013;2013:816236

27 O'Reilly MW, Sexton DJ, Dennedy MC, et al. Radiological remission and recovery of thirst appreciation after infliximab therapy in adipsic diabetes insipidus secondary to neurosarcoidosis. QJM 2013

28 Tanaka K, Yamamoto M, Okazaki K, Yamaguchi T, Sugimoto T. Partial improvement of anterior pituitary deficiency following steroid treatment in a patient with neurosarcoidosis accompanied by central diabetes insipidus. Intern Med 2012;51(16):2175-2179

29 Langrand C, Bihan H, Raverot G, et al. Hypothalamo-pituitary sarcoidosis: a multicenter study of 24 patients. QJM 2012; 105(10):981-995

30 Tedford M, Horine L, Burns ML. The resolution of pituitary mass in neurosarcoidosis. J Ark Med Soc 2012;108(10):226-228

31 Titlić M, Dolić K, Besenski N. Mild cognitive disorder as clinical manifestation of pituitary stalk neurosarcoidosis: case report. Acta Clin Croat 2011;50(4):581-587

32 Skowasch D, Pabst S, Wilhelm K, Grohé C. Diabetes insipidus due to neurosarcoidosis [in German]. Pneumologie 2011;65(8): 496-497

33 Alam T, Thomas S. Diabetes insipidus secondary to sarcoidosis presenting with caseating granuloma. BMJ Case Rep 2011:2011

34 Jomaa R, Sfar MH, Mhenni SY, et al. Isolated neurosarcoidosis revealed by diabetes insipidus, visual loss and diplopia in a child patient: a diagnostic problem. Clin Pediatr Endocrinol 2009;18(1): 51-54

35 Sundaresan P, Jayamohan J. Stereotactic radiotherapy for the treatment of neurosarcoidosis involving the pituitary gland and hypothalamus. J Med Imaging Radiat Oncol 2008;52(6): 622-626

36 Shimaoka Y, Tajima S, Koshio N, et al. Case of central neurosarcoidosis with panhypopituitalism [in Japanese]. Nihon Kokyuki Gakkai Zasshi 2008;46(10):814-819

37 Hasumi Y, Ishihara M, Asukata Y, et al. Case of neurosarcoidosis with rapid visual field defect progression [in Japanese]. Nippon Ganka Gakkai Zasshi 2007;111(9):728-734 